\title{
Atrial fibrillation had a negative impact on quality of life, and treatment with an implantable cardioverter defibrillator with atrial therapies helped patients regain a sense of normalcy
}

Deaton C, Dunbar SB, Moloney M, et al. Patient experiences with atrial fibrillation and treatment with implantable atrial defibrillation therapy. Heart Lung 2003;32:291-9.

\section{What are patients' experiences of living with symptomatic, drug refractory atrial fibrillation (AF) and treatment with an implantable cardioverter defibrillator with atrial therapies (ICD-AT)?}

\section{DESIGN}

Descriptive qualitative approach.

\section{SETTING}

3 clinical centres in the USA.

\section{PATIENTS}

11 patients (mean age $63 \mathrm{y}$, age range $35-80 \mathrm{y}, 73 \%$ men) who had AF that was unresponsive to treatment for $1-20$ years. Patients had been living with an ICD-AT device for a period of 6 months to 2 years. Patients had no documented ventricular arrhythmia.

\section{METHODS}

Patients participated in semistructured interviews, which were audiotaped and transcribed verbatim. 3 researchers coded the data according to key findings and themes using a qualitative interpretive approach. Codes were compared, and discrepancies were settled by a consensus process.

\section{MAIN FINDINGS}

Patients described a chronological course of AF illness and treatment, describing the early history of their illness, treatment seeking experiences, decision making process for seeking ICD-AT implantation, and adjustment to living with an ICD-AT. Patients' early experiences focused on the process of seeking a diagnosis and treatment plan. They described their experiences with AF, including misdiagnosis, minimisation, and poor treatment of AF by various providers; distressing experiences caused by frequent and intense AF symptoms, such as rapid heart rates, palpitations, dizziness, fatigue, anxiety, weakness, and shortness of breath; severe limitations in activities of daily living; distress caused by enduring previous treatment; and the continuous pursuit of successful treatment and attempts to maintain normalcy in daily life. The decision making process for seeking ICD-AT therapy included weighing symptom or treatment distress against anticipated risks or benefits, experiencing feelings of hope for better outcomes, and facing a lack of options.

Patients described their experiences of living with the ICD-AT, such as positive perceptions of the device because of AF symptom relief, ability to resume normalcy, and increased medication tolerance. Patients also described the need to incorporate the shock experiences (which were anxiety provoking and unpleasant) into their life routines. Patients described having gained insight into how

For correspondence: $\mathrm{Dr} \mathrm{C}$ Deaton, School of Nursing, Midwifery and Health Visiting, University of Manchester, Manchester, UK. Mary.C.Deaton@ man.ac.uk

Source of funding: Medtronic Inc, Minneapolis, Minnesota. to live with an ICD-AT and made suggestions to others in similar circumstances about preparation (eg, having a positive mental attitude) and seeking social support from others.

\section{CONCLUSIONS}

Patients felt that living with symptomatic, drug refractory atrial fibrillation was distressing and had a major negative impact on overall quality of life. They felt that treatment with an implantable cardioverter defibrillator with atrial therapies reduced their symptom distress and increased a sense of normalcy in daily activities.

\section{Commentary}

Trace he qualitative study by Deaton et al was done with a subset of patients from a larger quantitative study that examined patient acceptance and tolerance of new ICD technology. 'These descriptive findings highlight important clinical implications based on patients' experiences of technology adoption and their recommendations for care. In contrast to patients receiving ICD therapies for ventricular arrhythmias, AF is not life threatening. Patients in this study described ICD for refractory AF as life enhancing rather than life saving, with dramatic effects on their quality of life (eg, decreased symptom intensity, increased activity tolerance, and interest in health maintenance). Decisions to have this therapy typically occurred after numerous failures. Participants had experienced repeated trials of ineffective medications and numerous external cardioversions before ICD implantation. Ineffective advice and follow up from physicians led some patients to believe that healthcare providers had given up on them. ICD implantation was described as "the end of the road" and a "last resort" option.

Because AF does not need to be urgently converted to sinus rhythm, ICD-AT allows patients more control over activation of the device. After $A F$ is verified, shocks can either be automatically programmed to occur at a specific time (usually early morning) or activated by patients at a time and location of their choosing. Similar to other ICD therapies, participants reported great anxiety about the anticipation of the shock. The possibility of incorporating the shock into the patient's everyday routine requires careful coaching and teaching to anticipate individual responses and preferences. Social support was seen as a key factor in adapting to this technology. Patients recommended support groups or contact with others who have had similar experiences and preparation of family members to consider ways that they can be supportive and manage their own anxiety about the device.

Dianne M Tapp, RN, PhD Humeira Dhanji, RN, BN, MN student Faculty of Nursing, University of Calgary Calgary, Alberta, Canada

1 Sears SF, Burns J, Sotile R, et al. Do patients accept implantable atrial defibrillation therapy? Results from the patient atrial shock survey of acceptance and tolerance study. PACE 2002;24. 